\title{
Metformin promotes susceptibility to experimental Leishmania braziliensis infection
}

\author{
Filipe Rocha Lima ${ }^{1,4} /{ }^{+}$, Lais de Melo Ferreira ${ }^{1,5}$, Tainá Alves Malta ${ }^{1,5}$, \\ Icaro Bonyek-Silva ${ }^{2,4}$, Reinan Lima Santos ${ }^{2,4}$, Natália Machado Tavares ${ }^{2}$, \\ Edgar Marcelino de Carvalho Filho ${ }^{3,4}$, Sérgio Arruda ${ }^{1,5}$
}

${ }^{1}$ Fundação Oswaldo Cruz-Fiocruz, Instituto Gonçalo Moniz, Laboratório Avançado de Saúde Pública, Salvador, BA, Brasil

${ }^{2}$ Fundação Oswaldo Cruz-Fiocruz, Instituto Gonçalo Moniz, Laboratório de Interação Parasito-Hospedeiro e Epidemiologia, Salvador, BA, Brasil

${ }^{3}$ Fundação Oswaldo Cruz-Fiocruz, Instituto Gonçalo Moniz, Laboratório de Pesquisa Clínica, Salvador, BA, Brasil

${ }^{4}$ Universidade Federal da Bahia, Salvador, BA, Brasil

${ }^{5}$ Universidade Estadual da Bahia, Departamento de Ciências da Vida, Salvador, BA, Brasil

BACKGROUND Metformin (MET) is a hypoglycemic drug used for the treatment of diabetes, despite interference in host immunity against microorganisms. Cutaneous infection caused by pathogens such as Leishmania braziliensis (Lb), the agent responsible for cutaneous leishmaniasis (CL) in Brazil, represents an interesting model in which to evaluate the effects associated with MET.

OBJECTIVE To evaluate the modulatory effect of MET in $L b$ infection.

MATERIAL AND METHODS Experimental study of $L b$ infection and MET treatment in BALB/c mice and Raw 264.7 macrophages.

FINDINGS MET treatment interfered with lesion kinetics, increased parasite load and reduced macrophage proliferation. Low concentrations of MET in $L b$ culture allow for the maintenance of stationary parasite growth phase. $L b$-infected cells treated with MET exhibited increased parasite load. While both MET and $L b$ infection alone promoted the production of intracellular reactive oxygen species (ROS), reduced levels of ROS were seen in MET-treated $L b$-infected macrophages.

MAIN CONCLUSION Experimental treatment with MET interfered with the kinetics of cutaneous ulceration, increased $L b$ parasite load, altered ROS production and modulated cellular proliferation. Our experimental results indicate that MET interfere with the evolution of CL.

Key words: metformin - cutaneous leishmaniasis - immunomodulation - susceptibility - infection

Cutaneous leishmaniasis (CL) is a clinical form of the anthropozoonotic disease complex caused by protozoa of the genus Leishmania.(1) In Brazil, CL is commonly caused by Leishmania braziliensis $(L b)$ and is clinically characterised by one or more oval-shaped ulcerative lesions, or rounded, granulomatous-bottomed ulcerations with well-defined raised borders. ${ }^{(2,3)}$ The low parasitic burden found in lesions results from a Th1-mediated intense inflammatory response, in addition to cytokine production, such as IFN- $\gamma$, TNF, IL-12 and IL-2. Intracellular death in these parasites results from the microbicidal action of IFN- $\gamma$-stimulated macrophages. However, exacerbated inflammation provokes tissue damage and initiates a pathological process culminating in the formation of ulcers on the skin of the host. ${ }^{(3,4,5)}$

In Brazil, pentavalent antimonials $\left(\mathrm{Sb}^{+5}\right)$ are the firstline drugs for $L b$ infection treatment. Despite high efficacy, the numerous side effects, such as cardiac, renal and hepatic toxicity, induced by these drugs consequently

doi: 10.1590/0074-02760200272

Financial support: CAPES (Finance Code 001), CNPq, FAPESB.

+ Corresponding author: rfilipelima@gmail.com

(D) https://orcid.org/0000-0001-7629-4963

Received 27 May 2020

Accepted 03 November 2020 restricts use to certain groups of patients. ${ }^{(2)}$ In addition, recent studies have demonstrated increasing resistance to these antimonials in several Latin American countries, as well as in India. ${ }^{(6,7,8)}$ In light of this scenario, several studies have investigated alternative treatment strategies, such as combined therapy involving $\mathrm{Sb}^{+5}$ and immunomodulators, such as pentoxifylline, an inhibitor of TNF production, which seemed to accelerate cure in patients. ${ }^{(1,9)}$

The immunomodulatory effects of antidiabetic drugs, such as metformin (MET), has been described in cancer and infectious diseases, including tuberculosis and pneumonia caused by Legionella pneumophila. ${ }^{(10,11)}$ Antidiabetic drug-induced effects include 5' AMP-activated protein kinase (AMPK) activation, signal transducer and activator of transcription 3 (STAT3) inhibition, and the increased production of mitochondrial reactive oxygen species (ROS). ${ }^{(12,13)}$ Although described in other contexts, little is known about the action of MET in parasitic infections, such as $L b$ Considering the high prevalence of CL in Brazil, together with this drug's ample availability and low cost, the present study aimed to analyse the effects of MET on the immune response against $L b$ in an experimental mouse model.

\section{MATERIALS AND METHODS}

Experimental model design - After receiving ethical approval by the local Institutional Review Board for Animal Experimentation (protocol 013/2017), 6-weekold male isogenic BALB/c mice were obtained from the 
Animal Care Facility of the Instituto Gonçalo Moniz, Fundação Oswaldo Cruz (IGM-Fiocruz) in Salvador, Bahia, and maintained under controlled conditions (temperature $22 \pm 2^{\circ} \mathrm{C}, 50 \%$ humidity, 12-hour light/ dark cycles). Mice were divided into three groups of five according to MET treatment and $L b$ infection status. Group 1: $L b$ infection; Group 2: MET treatment alone; Group 3: $L b$ infection and MET treatment.

Metformin hydrochloride treatment - Mice from Groups 2 and 3 received continuous pre-treatment for six weeks (prior to infection procedures) with an oral solution containing metformin hydrochloride $(\mathrm{Hcl})(\mathrm{Ga}-$ lena Biopharma, India) at a dosage of $500 \mathrm{mg} / \mathrm{kg}$ diluted in water. Following $L b$ infection procedures, mice in Groups 2 and 3 continued to receive MET solution orally for an additional six weeks.

Leishmania braziliensis infection - Groups 1 and 3 were intradermally infected in the left ear using a $30 \mathrm{G}$ needle syringe containing $10 \mu \mathrm{L}$ of $2 \times 10^{5}$ stationaryphase metacyclic $L b$ promastigotes (strain MHOM/ BR/01/BA788). Parasites were cultivated in Schneider's medium (Sigma Chemical Co., St Louis, MO, USA), supplemented with $20 \%$ inactivated foetal bovine serum (FBS) (Gibco, USA), L-glutamine (2 $\mathrm{mM})$, penicillin $(100 \mathrm{U} / \mathrm{mL})$ and streptomycin $(100 \mathrm{mg} / \mathrm{mL})$, for 5-7 days under $23^{\circ} \mathrm{C}$ until reaching stationary phase.

The diameter of the infected ear was measured weekly in Groups 1 and 3 with the aid of a digital caliper. Measurements were expressed in millimeters $(\mathrm{mm})$ and used to compare between groups.

Parasite load quantification - Parasitic load was determined in Groups 1 and 3 based on a limiting dilution assay ${ }^{(14)}$ at six weeks after infection, when the animals were euthanised and their ipsilateral lymph nodes and infected ears were removed. Following extraction under sterile conditions, the obtained mouse organs were macerated using a $40 \mu \mathrm{m}$ pore size cell strainer, with the addition of $2 \mathrm{~mL}$ Roswell Park Memorial Institute (RPMI) medium supplemented with $10 \%$ FBS, L-glutamine (2 $\mathrm{mM})$, penicillin $(100 \mathrm{U} / \mathrm{mL})$ and streptomycin $(100 \mathrm{mg}$ $/ \mathrm{mL}$ ). Collected cells were then centrifuged at $1500 \mathrm{rpm}$ under $4^{\circ} \mathrm{C}$ for $10 \mathrm{~min}$. The precipitate was resuspended in $1 \mathrm{~mL}$ of supplemented Schneider medium; $20 \mu \mathrm{L}$ aliquots were serially diluted on a 96-well flat-bottomed plate containing $180 \mu \mathrm{L} /$ well of supplemented Schneider medium. Eight serial dilutions were performed in triplicate. Plates were sealed and incubated in a Biochemical Oxygen Demand (BOD) incubator at $23^{\circ} \mathrm{C}$ for 10 days. Wells were observed under an inverted optical microscope (Nikon) at $20 \times$ magnification, with daily readings taken beginning on the third day of cultivation to register viable promastigotes.

Cell viability testing - Raw 264.7 macrophages were plated at a concentration of $10^{5}$ cells/well on a CELLSTAR $^{\circledR}$ 96-well flat plate containing $200 \mu \mathrm{L} /$ well of Dulbecco's Modified Eagle's medium (DMEM) (GibcoTM) supplemented with 10\% FBS (Gibco), L-glutamine $(2 \mathrm{mM})$, penicillin $(100 \mathrm{U} / \mathrm{mL})$ and streptomycin $(100 \mathrm{mg} / \mathrm{mL})$. Experiments were performed in triplicate under the following conditions: positive control (viable cells in DMEM medium), negative control [cells grown in 1x phosphate-buffered saline (PBS)], $\mathrm{R}$ white control (DMEM medium) and macrophages incubated with 2, 2.5, 5 and $10 \mathrm{mM}$ of MET hydrochloride for $24 \mathrm{~h}$ at $37^{\circ} \mathrm{C}$ under $5 \% \mathrm{CO}_{2}$. Next, $20 \mu \mathrm{L}$ from each well was removed, followed by the addition of $20 \mu \mathrm{L}$ of Alamarblue ${ }^{\mathrm{TM}}$ Cell Viability Reagent (ThermoFisher Scientific), followed by a 4-hour reincubation period under the conditions described above in the absence of light. Readings were performed on a spectrophotometer at wavelengths of $570 \mathrm{~nm}$ and $600 \mathrm{~nm}$, with results interpreted following the manufacturer's protocol. The selected concentration for subsequent experimentation was determined to be $2 \mathrm{mM}$ of MET.

Macrophage viability assays - Cell growth kinetics were investigated using a 24-well plate assay (CELL$\mathrm{STAR}^{\circledR}$ ) performed in triplicate with $10^{5}$ macrophages/ well under the following conditions: 1) no MET; 2) treatment with $2 \mathrm{mM}$ MET, followed by incubation; 3) cells pre-treated with $2 \mathrm{mM}$ of MET six days prior to receiving an additional $2 \mathrm{mM}$ MET, followed by incubation. Counts were performed at $24,48,72$ and $96 \mathrm{~h}$ of cultivation at $37^{\circ} \mathrm{C}$ under $5 \% \mathrm{CO}_{2}$ in supplemented DMEM $(1 \mathrm{~mL})$ (GibcoTM). At each cell counting timepoint, DMEM medium was discarded and cells were removed by enzymatic action using $1 \mathrm{~mL} 0.25 \%$ trypsin-EDTA (Sigma-Aldrich) for $1 \mathrm{~min}$ at $37^{\circ} \mathrm{C}$ under mechanical homogenisation with a micropipette. The aspirated volume $(1 \mathrm{~mL})$ was inactivated with $9 \mathrm{~mL}$ supplemented DMEM and centrifuged at $1200 \mathrm{rpm}$ under $4^{\circ} \mathrm{C}$ for $10 \mathrm{~min}$. The medium was discarded and obtained cellular pellet was resuspended in 1 $\mathrm{mL}$ of supplemented DMEM. Trypan Blue (Sigma-Aldrich) was added to the cell suspension aliquots at a ratio of 1:10. Cell viability was then assessed by counting in a Neubauer chamber at 400x magnification.

Influence of metformin on L. braziliensis growth Metacyclic $L b$ promastigotes ( $5 \times 10^{5} / \mathrm{mL}$ ) were placed in $25 \mathrm{~cm}^{2}$ cell culture flasks (CELLSTAR®) containing 5 $\mathrm{mL}$ of supplemented Schneider's medium and grown in a BOD at $23^{\circ} \mathrm{C}$. Cultivation was performed in duplicate under the following conditions: $0,2,5$ or $10 \mathrm{mM}$ of MET. Promastigote growth was measured via daily counts for six days by removing $10 \mu \mathrm{L}$ from culture flasks, which was placed in a Neubauer chamber and observed under an E200 (Nikon) optical microscope at 400x magnification.

Raw 264.7 macrophage infection with L. braziliensis - Macrophages were plated at a concentration of $10^{5}$ cells/well on 24-well plates (CELLSTAR ${ }^{\circledR}$ ) in $300 \mu \mathrm{L}$ of supplemented DMEM medium, followed by the addition of circular coverslips $(13 \mathrm{~mm})$ for macrophage adhesion.

Cells were cultivated under the following conditions: 1) negative control without treatment or infection; 2) positive control with $100 \mathrm{ng} / \mathrm{mL}$ lipopolysaccharide (LPS) treatment; 3) $2 \mathrm{mM}$ MET treatment without infection; 4) infection with $L b$; 5) infection with $L b$ and 2 mM MET treatment; 6) $100 \mathrm{ng} / \mathrm{mL}$ LPS and $2 \mathrm{mM}$ MET treatment; 7) infection with $L b, 100 \mathrm{ng} / \mathrm{mL}$ LPS and 2 mM MET treatment. Cells under conditions 3, 5, 6 and 7 were pretreated with $2 \mathrm{mM}$ MET for five days prior to 
challenge, and received an additional $2 \mathrm{mM}$ MET following infection procedures. The multiplicity of infection (MOI) used was 10:1 parasites/macrophage, with a 4-hour incubation at $37^{\circ} \mathrm{C}$ under $5 \% \mathrm{CO}_{2}$. Experimental conditions were performed in quadruplicate and the experiment was repeated twice.

Parasite infection rate and quantification - Coverslips were washed thrice with $1 \mathrm{~mL}$ PBS $1 \mathrm{x}$ and then subjected to rapid panoptic staining (Laborclin). Rapid panotic number $1(300 \mu \mathrm{L})$ was added to the wells and stored for $10 \mathrm{~min}$ in a refrigerator at 2 to $8^{\circ} \mathrm{C}$, followed by $300 \mu \mathrm{L}$ of panoptic number 2 for 1 minute at RT, $300 \mu \mathrm{L}$ of panoptic number 3 for 10 seconds at RT and finally washed thrice with $1 \mathrm{~mL}$ of distilled water with the aid of a $1000 \mu \mathrm{L}$ micropipette during the entire process. The coverslips were then mounted using Entellan (Sigma-Aldrich) on 26x76 $\mathrm{mm}$ glass slides for further evaluation under an optical microscope (Nikon) at 100x magnification. The number of infected cells (presence of parasites in intracellular medium) was totaled out of 100 counted cells, with values expressed as percentages, and the total number of parasites was counted inside infected cells.

Evaluation of intracellular parasite viability - After $4 \mathrm{~h}$ of infection, the wells were washed thrice with $300 \mu \mathrm{L} 0.9 \% \mathrm{NaCl}$ solution at $37^{\circ} \mathrm{C}$ to remove any noninternalised promastigotes. Prior to washing, $200 \mu \mathrm{L} /$ well of supplemented Schneider medium was added and the sealed plate was incubated in a BOD at $23^{\circ} \mathrm{C}$ for 96 $\mathrm{h}$. The number of promastigotes in $10 \mu \mathrm{L}$ aliquots were counted in a Neubauer chamber at $400 \mathrm{x}$ magnification.

ROS quantification in culture supernatant - Culture supernatant was distributed on two 96-well flat-bottomed plates (Greiner Bio-One) in triplicate. To measure ROS in the supernatant, an alternative methodology involving Griess reaction (Sigma-Aldrich) was used. The Griess method allows, via colorimetric reaction, the measurement of nitric oxide (NO) production through nitrite conversion using spectrophotometry. Two experimental conditions were used: wells in one plate received the addition of hydroxylamine $\left(\mathrm{NH}_{2} \mathrm{OH}\right)$, while another did not. Optical density (OD) values were transformed in concentrations $(\mu \mathrm{M})$ by measuring differences in OD readings between the two plates, thus allowing for an indirect evaluation of ROS in the culture supernatant, due to the inhibition of the nitrification reaction via the presence of the $\mathrm{NH}_{2} \mathrm{OH}$ intermediate substrate.

Quantification of intracellular ROS - Cells were submitted to a CellROX Oxidative Stress Reagent assay (ThermoFisher Scientific). All wells containing macrophages were washed thrice with $1 \mathrm{x}$ PBS, and $300 \mu \mathrm{L} /$ well of supplemented DMEM medium and CellROX reagent were added. After being incubated in the dark at $37^{\circ} \mathrm{C}$ under $5 \% \mathrm{CO}_{2}$ for $30 \mathrm{~min}$, wells were washed three times again with $1 \mathrm{x}$ PBS, and the cell coverslips were fixed with $3.7 \%$ paraformaldehyde (PFA) for $15 \mathrm{~min}$, then mounted on a $23 \times 76 \mathrm{~mm}$ glass slide with $5 \mu \mathrm{L}$ of 4', 6-diamino-2- phenyl indole (DAPI) in antifade mounting medium (ThermoFisher Scientific). Subsequently, the slides were analysed under an inverted fluorescence microscope (Leica) in the range of $485 / 520 \mathrm{~nm}$, and images of fields containing eight cells each were captured to quantify the corrected total fluorescence coefficient (CTCF) using OlyVIA 7.0 software (Olympus). This analytic strategy consists of measuring fluorescence in the area occupied by a cell, averaging values from the eight cells analysed, and then subtracting the fluorescence background average from five extracellular spaces.

Statistical analysis - The D'Agostino test was used to classify the distribution of samples as parametric or nonparametric. The Mann-Witnney or Kruskal-Wallis tests were used for nonparametric samples, while the t-test or one way analysis of variance (ANOVA) was employed for parametric distribution. The Chi-square test for trend was also used. Analyses were performed using GraphPad Prism v.7 software (GraphPad Software ${ }^{\circledR}$, San Diego, $\mathrm{CA}$ ), and $\mathrm{p}$ values less than or equal to 0.05 were considered significant.

\section{RESULTS}

MET delays the appearance of Leishmania papules and modifies lesion size - Three weeks after inoculating metacyclic promastigotes in stationary phase, G1 $(L b)$ animals presented a $20 \%$ larger ear thickness compared to $\mathrm{G} 3(\mathrm{Lb}+\mathrm{MET})$ : mean $0.7 \mathrm{~mm}( \pm 0.07 ; \mathrm{p}=0.0048)$, small ulcerated papule with acneiform aspect and cutaneous hyperemia. On the other hand, lesion size in G3 was $3.4 \%$ smaller than G2 (control) of $0.56 \mathrm{~mm}$ ( \pm 0.055 ), ie, showing no evident tissue damage. In the 4 th week of infection, lesion size in G1 decreased by $5.7 \%$, while group G3 increased by $15.1 \%$, demonstrating the appearance of acneiform papules without ulceration. At six weeks after infection, the absence of ulcers was seen in non-MET (G1) animals, with ear thickness averaging $0.58 \mathrm{~mm}( \pm 0.084)$, just $0.8 \%$ different from $\mathrm{G} 2$, the uninfected group (Fig. 1A-B).

MET promotes increased parasitic load in lesions and ipsilateral lymph node - Six weeks after infection, all animals were euthanised and the left ears and cervical lymph nodes were resected in G1 $(\mathrm{n}=05)$ and G3 (n $=05$ ). Mouse organs were macerated and then cultivated in serial dilutions for parasitic load measurement. At the site of infection (Fig. 1C) and in the cervical lymph nodes (Fig. 1D), G3 presented the highest limiting dilution positivity $\left(5 \times 10^{6}\right.$ parasites $\left./ \mathrm{mL}\right), 10^{3}$ more promastigotes compared to $\mathrm{G1}(\mathrm{p}=0.02 ; \mathrm{p}=0.01$, respectively).

MET exerts no cytotoxic effects and inhibits Raw 264.7 macrophage growth - Cell viability following exposure to different MET concentrations was evaluated in cultures of Raw 264.7 macrophages, with an absence of any observable cytotoxic effects arising due to MET within $24 \mathrm{~h}$. Cell viability values ranged from 85 to $100 \%$ relative to the positive control, i.e. cells that did not receive MET in supplemented DMEM medium.

Using the selected concentration for subsequent experimentation was determined to be $2 \mathrm{mM}$ of MET, a cell viability assay $\left(1 \times 10^{5}\right.$ cells/well $)$ was performed to quantify the number of viable cells after $24,48,72$ and $96 \mathrm{~h}$ of MET exposure by comparing cells pretreated for 
A

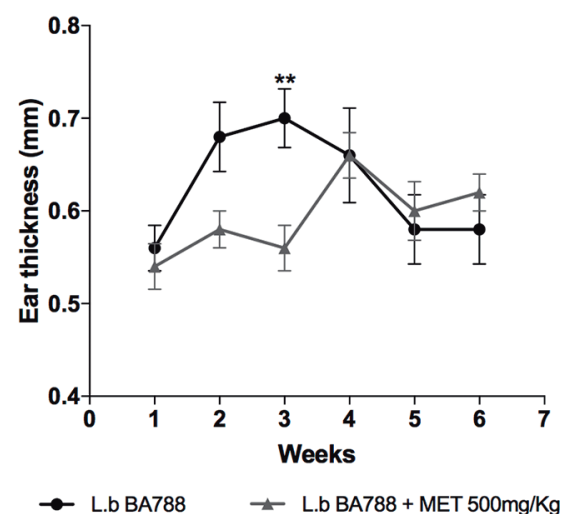

C

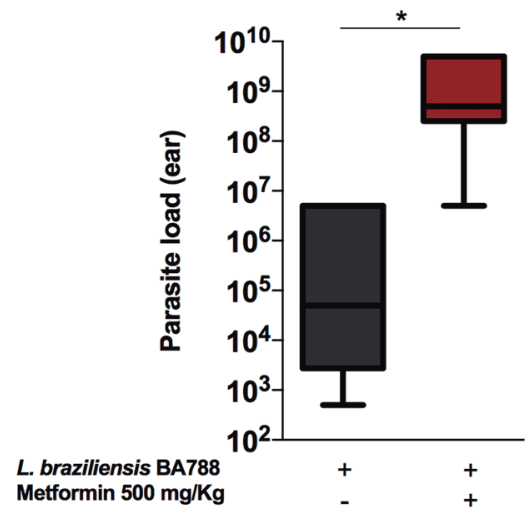

B

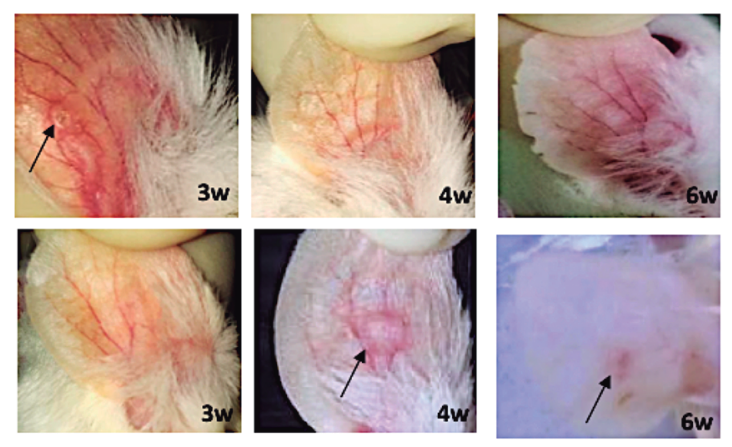

D

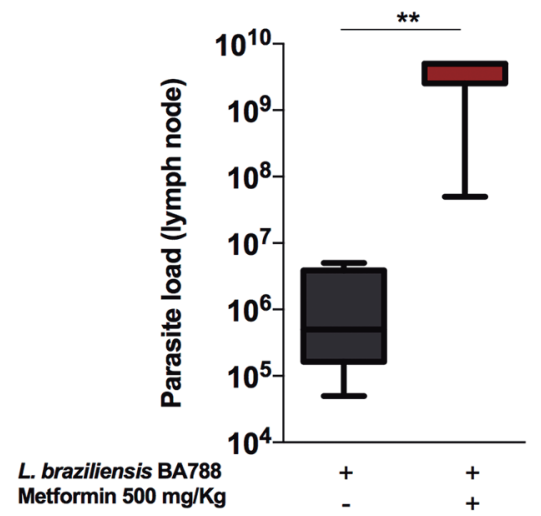

Fig. 1: monitoring of skin lesion appearance and parasite load in an experimental model of infection with Leishmania braziliensis (Lb). Metformin (MET)-treated mice received the first dose $(500 \mathrm{mg} / \mathrm{kg})$ six weeks before infection, and continued treatment at the same dosage for six additional weeks after infection. The untreated group had no contact with MET at any time during experimentation. Graph of lesion size in infected animals measured at the site of infection, the left ear, with the aid of a digital caliper (A). Photographic documentation of the left ear of the group infected with $L b$ and not treated with MET (upper panels) and mice infected with $L b$ and treated with MET (lower panels), during the third ( $3 \mathrm{w})$, fourth $(4 \mathrm{w})$ and sixth weeks ( $6 \mathrm{w})$ post-infection (B). Comparisons of parasite load between groups infected with $L b$ and not treated with MET versus $L b$-infected and MET-treated in the left ear $(C)$ and lymph node (D) six weeks post-infection, measured by limiting dilution assay. Statistical analysis performed using one-way ANOVA (A) and Mann-Whitney test (C-D). Asterisks indicate significant differences, respectively $\left(* * \mathrm{p}=0.0048 ;{ }^{*} \mathrm{p}=0.02 ; * * \mathrm{p}=0.01\right)$.

six days in MET and those without previous drug exposure. After $96 \mathrm{~h}$, cultured cells without prior exposure to MET achieved an average growth of $1.4 \times 10^{5}$ cells, i.e. $22.5 \%$ higher than pretreated cells. Cultures without MET yielded an average of $5.9 \times 10^{5}$ cells/well after 96 $\mathrm{h}$, representing a $76.3 \%$ higher proliferation rate compared to macrophages not previously exposed to MET $(p=0.0024)$, versus $81.3 \%$ more compared to previously treated cells $(\mathrm{p}=0.0016)($ Fig. 2A).

Low MET concentrations increase Lb promastigote growth - After verifying that MET interferes with macrophage growth in vitro, we verified the effect of this drug on $L b$ promastigote cultivation in vitro by growing $5 \times 10^{5}$ metacyclic promastigotes $/ \mathrm{mL}$ in $5 \mathrm{~mL}$ of DMEM. Cultivation was quantified daily for six days until proliferation was observed to decrease. On the 6th day of culture, $34.3 \%$ higher growth was seen in cells receiving $2 \mathrm{mM}$ MET compared to those that did not receive the drug, with an average of $1.7 \times 10^{8}$ parasites $/ \mathrm{mL}$ and demonstrated continuance of in log phase growth. Under culture conditions involving $5 \mathrm{mM}$ MET, no difference was seen compared to drug-free culture (average of $6 \mathrm{x}$ $10^{7}$ parasites $/ \mathrm{mL}$ ). Using $10 \mathrm{mM} \mathrm{MET,} 8.5 \%$ less proliferation was observed compared to drug-free culture (average of $5.5 \times 10^{7}$ parasites $/ \mathrm{mL}$ ) (Fig. 2B).

In vitro MET treatment resulted in increased infection rate and parasitic load in Raw macrophages 264.7 - After a 4-hour infection period, the percentage of infected cells (Fig. 3A) and the number of parasites relative to the quantified cells (Fig. 3B) were counted. The addition of MET to cell cultures was found to interfere with the frequency of infected macrophages $(p=0.006)$ compared to untreated cells. The number of parasites/ cells was $46.8 \%$ higher in infected cells treated with MET compared to untreated cells $(\mathrm{p}=0.002)$.

Similar to our results regarding infection rate and parasites counts, intracellular parasitic viability analysis revealed an increase in parasite viability at $96 \mathrm{~h}$ in macrophages treated with MET compared untreated cells ( $\mathrm{p}$ $<0.0001$ ) (Fig. 3C). 
A

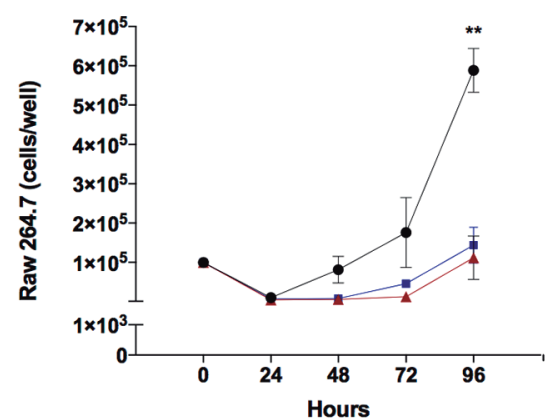

B

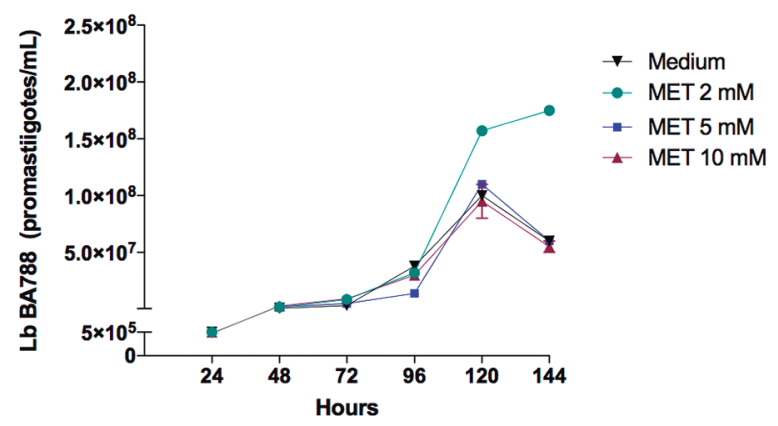

Fig. 2: cultivation of Raw 264.7 macrophages and Leishmania braziliensis $($ Lb) promastigotes exposed to metformin (MET). Cell growth kinetics investigated in triplicate assays $\left(10^{5}\right.$ cells/well) under the following conditions: no MET (Medium); treatment with 2 mM MET; cells pretreated with $2 \mathrm{mM}$ MET continuously for six days prior to receiving an additional $2 \mathrm{mM}$ MET. Counts performed at 24, 48, 72 and $96 \mathrm{~h}$ after cultivation (A). Metacyclic $L b$ promastigote $\left(5 \times 10^{5} / \mathrm{mL}\right)$ growth measured daily at $24,48,72,96,120,144 \mathrm{~h}$ under optical microscopy at 400x magnification, in response to the following conditions: 0, 2, 5 or $10 \mathrm{mM}$ of MET (B). Statistical analysis performed using one-way ANOVA (A). Asterisks indicate significant differences (Medium vs 2 mM MET; $p=0.0024$ ) and (Medium vs 2 mM P MET; $\mathrm{p}=0.0016$ ).
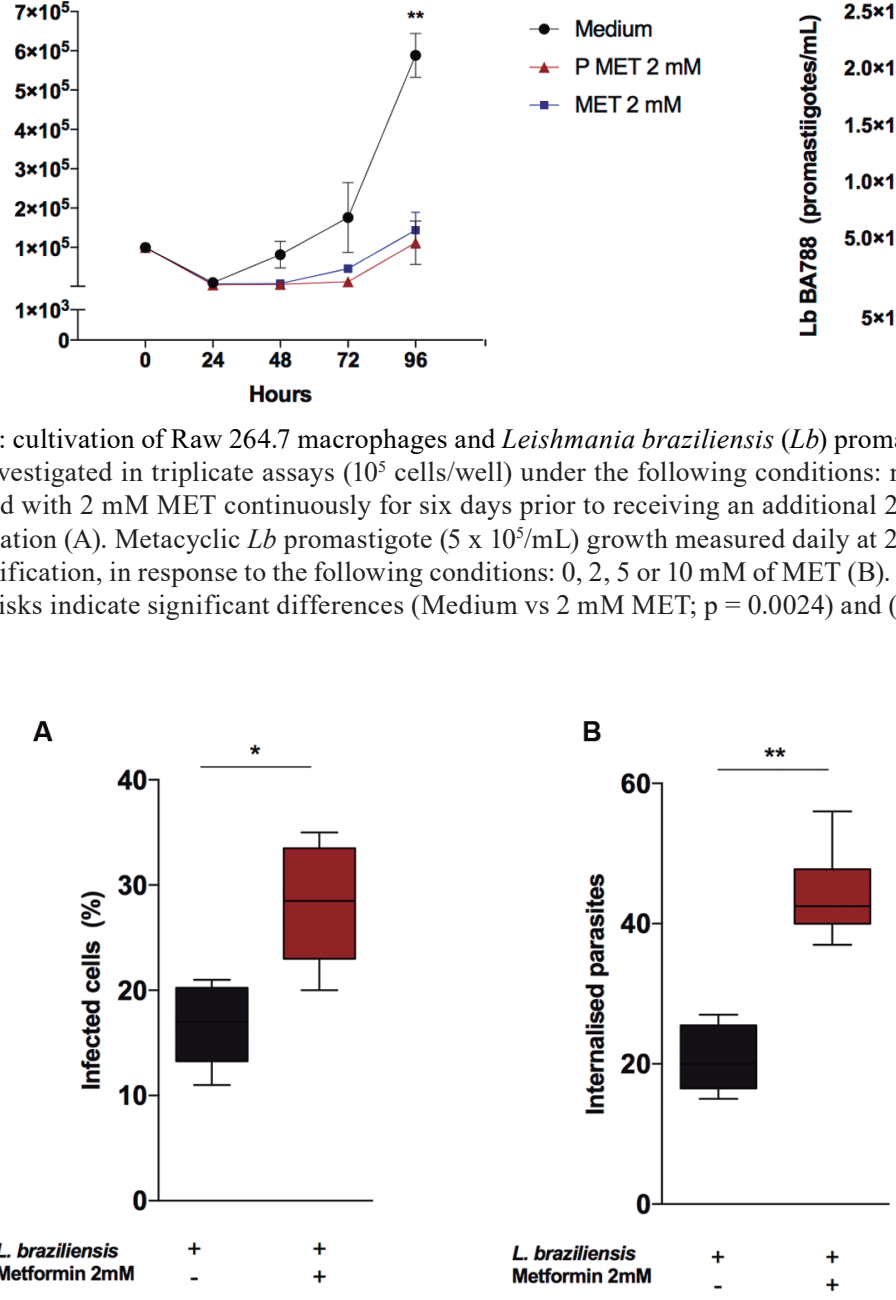

Fig. 3: comparative evaluation of parasitic load and intracellular viability of Leishmania braziliensis (Lb) in Metformin (MET)-treated and untreated Raw 264.7 macrophages ( $10^{5}$ cells/well). The MET group was previously treated with 2 mM MET for six days before challenge and received an additional $2 \mathrm{mM}$ after infection (MOI 10:1 parasites/macrophage). Graphical representation of infection rate in 100 cells, with results expressed as percentages (A), quantification of internalised parasites in infected cells by optical microscopy at 1000x magnification (B). Intracellular parasitic viability was evaluated after $96 \mathrm{~h}$ and promastigotes were counted in infected cells $(\mathrm{C})$. Statistical analysis was performed using Mann-Whitney test (A-B) and Chi-square for trend test $(\mathrm{C})$. Asterisks indicate significant differences $(* \mathrm{p}=0.006 ; * * \mathrm{p}=0.02)$.

Taken together, these data suggest that MET-treated cells may decrease the microbicidal potential of macrophages during $L b$ infection.

MET suppresses the release of ROS in the extracellular environment and intracellular ROS production in Lb-infected Raw 264.7 macrophages - ROS from macrophages was quantified in culture supernatants. After infection with $L b$, MET treatment suppressed ROS production by $89.6 \%$ compared to uninfected treated cells $(\mathrm{p}=0.007)$ (Fig. 4). In Fig. 5, representative microscopic fields illustrating corrected total cell fluorescence intensity allowed for the qualitative verification of fluorescence with respect to ROS (A), DAPI (B) and overlap (C) (Fig. 5A). Our results indicate a significant increase in ROS production in $L b$-infected cells, evidencing the effect of $L b$ on macrophage activation compared to uninfected cells $(\mathrm{p}<0.0001) . L b$ was found to induce a su- perior effect compared to stimulation with LPS $(p=0.01)$ or MET $(p=0.0008)$, and also resulted in higher ROS production compared to LPS-stimulated cells and treated with $\operatorname{MET}(\mathrm{p}=0.01)$. This analysis highlights differences in the production of reactive mediators compared to the negative control, under stimulation with MET treatment and $L b$ infection ( $\mathrm{p}=0.008)$, MET and LPS ( $\mathrm{p}=0.03)$ or MET, LPS and $L b(\mathrm{p}=0.006)$. Despite achieving statistical significance $(p=0.08)$, we also found that MET reduced intracellular ROS production by $34.1 \%$ in $L b$ infection compared untreated infected macrophages (Fig. 5B).

\section{DISCUSSION}

The present results demonstrate that MET treatment in $L b$-infected mice delays the appearance of papules characteristic of $L b$ infection; however, this drug not found to prevent ulceration. A study by Qing ${ }^{(15)}$ highlights the molecular mechanisms of MET in inducing the AMPK / 
mTOR axis by promoting NLRP3 inhibition as a crucial event in M2 macrophage induction, as well as promoting rapid tissue healing. Another study by Ochoa-Gonzalez ${ }^{(16)}$ stated that MET blocks mTOR action, shortens the cell cycle, reduces in vitro keratinocyte cell proliferation, negatively impacted healing in an experimental model and worsened the clinical condition of foot ulcers in diabetic patients, suggesting that MET interferes with tissue remodeling, lesion size and lesion healing time.

MET induces AMPK activation by modulating the mitochondrial ADP / ATP balance, resulting in the production of mROS in macrophages, which increases the microbicidal potential of these cells in the context of other pathogenic infections, such as Mycobacterium tuberculosis ${ }^{(17)}$ and L. pneumophila. ${ }^{(12)}$ Other studies have presented evidence that by interfering with mitochondrial metabolism and adaptive immunity in Plasmodium infections, MET could exert a protective effect in malaria. ${ }^{(18,19)}$ The use of MET treatment in the experimental murine $L b$ infection model employed herein resulted in a 1,000-fold increase in the number of parasites at the inoculation site and draining lymph nodes compared to untreated mice. These findings suggest that MET exacerbates the production of ROS, a marker of cellular oxidative stress, and results in increased susceptibility to $L b$ Novais et al. ${ }^{(20)}$ reported that although Leishmania parasites are sensitive to ROS, the respiratory explosion that occurs in macrophages that are not activated by adaptive immunity in response to infection is insufficient to enable parasite killing. Fukai et al. ${ }^{(21)}$ stated that some parasite species may induce antioxidant responses as an escape mechanism. Khouri et al. ${ }^{(22)}$ found significant increases in the expression and activity of superoxide desmutase-1 (SOD-1) occurring in human macrophages infected with L. amazonensis, which directly favors parasite survival.

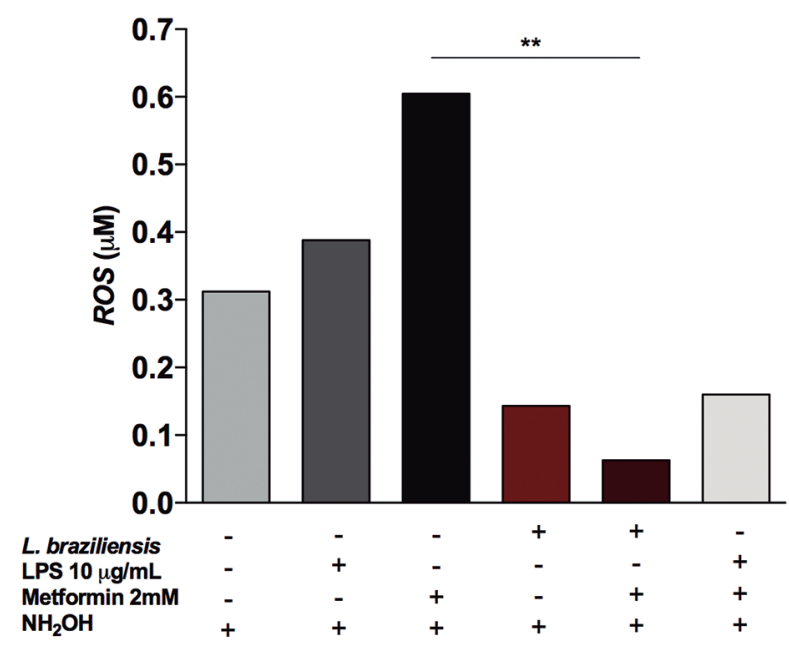

Fig. 4: production of reactive oxygen species (ROS) measured in the culture supernatant of infected Raw 264.7 macrophages $\left(10^{5} \mathrm{cells} /\right.$ well, multiplicity of infection (MOI) 10:1 parasites/macrophage) using an alternative methodology involving the Griess reaction. Optical density values were transformed into concentrations $(\mu M)$. Statistical analysis was performed using Kruskal-Wallis test. Asterisks indicate significant differences $(* * p=0.007)$. LPS: lipopolysaccharide. $\mathrm{NH} 2 \mathrm{OH}$ : hydroxylamine.
A

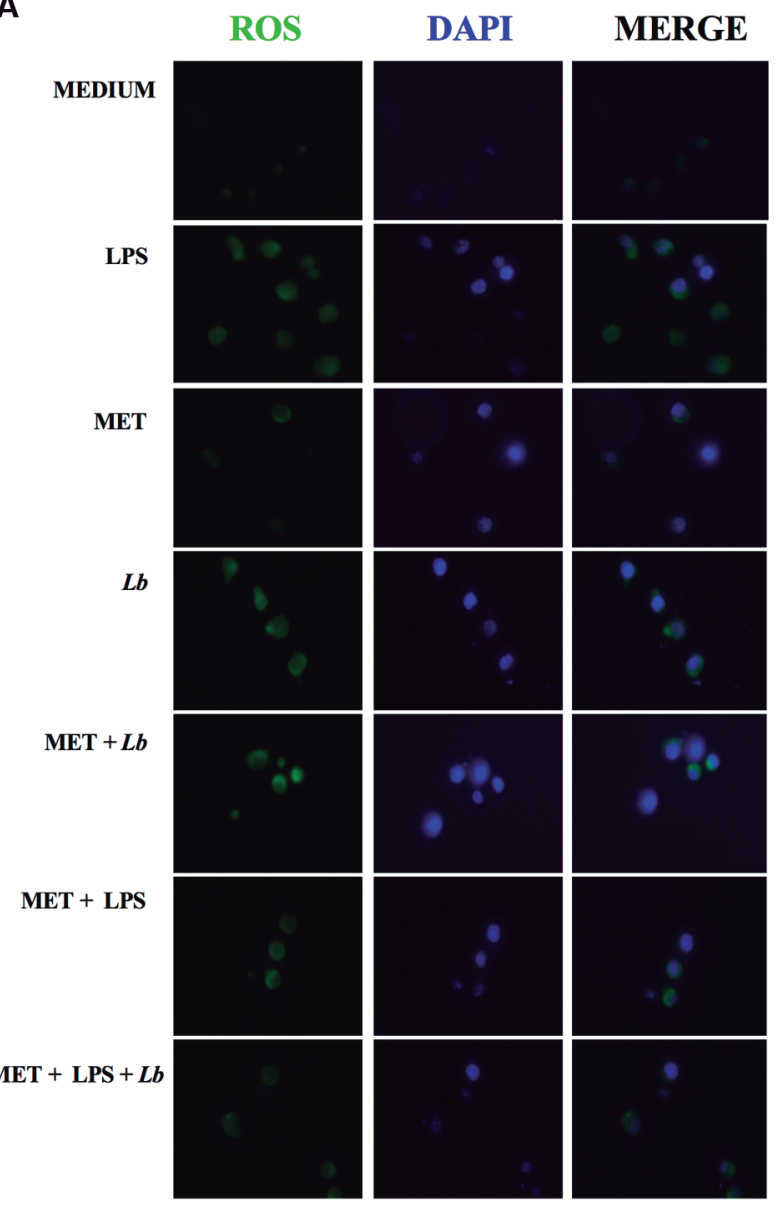

B

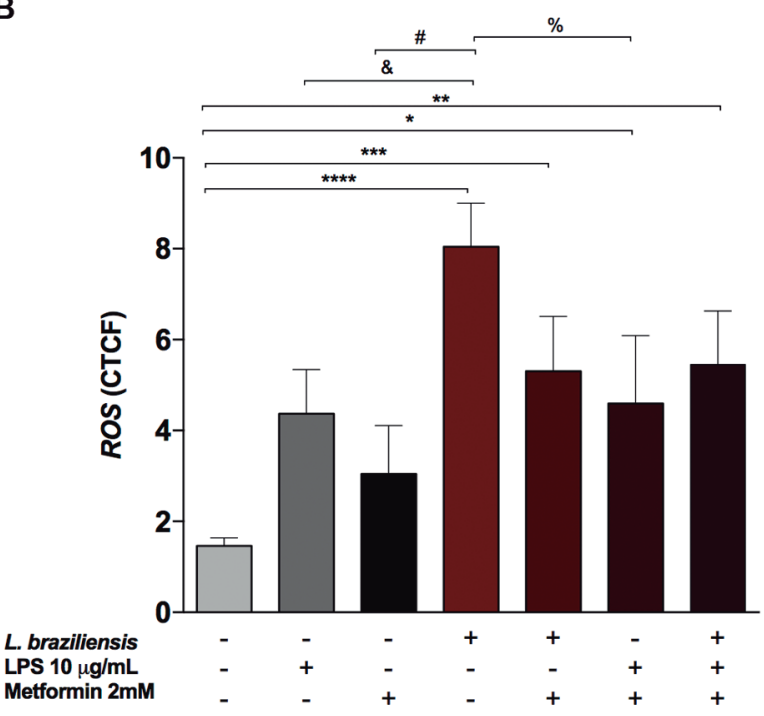

Fig. 5: intracellular quantification of reactive oxygen species (ROS) in Raw 264.7 macrophages ( $10^{5}$ cells) infected with Leishmania braziliensis ( $L b$ ) [multiplicity of infection (MOI) 10:1 parasites/macrophage] under different conditions. Representative images of cell labeling by immunofluorescence (A) and quantification of corrected total fluorescence coefficient (B). Statistical analysis performed using the one-way ANOVA. Symbols indicate significant differences $(* * * * \mathrm{p}=0.0001 ; * * * \mathrm{p}=0.008 ; * \mathrm{p}=0.03 ; * * \mathrm{p}=0.006 ; \& \mathrm{p}=0.01$ $\# p=0.0008 ; \% p=0.01$ ). LPS: lipopolysaccharide. 


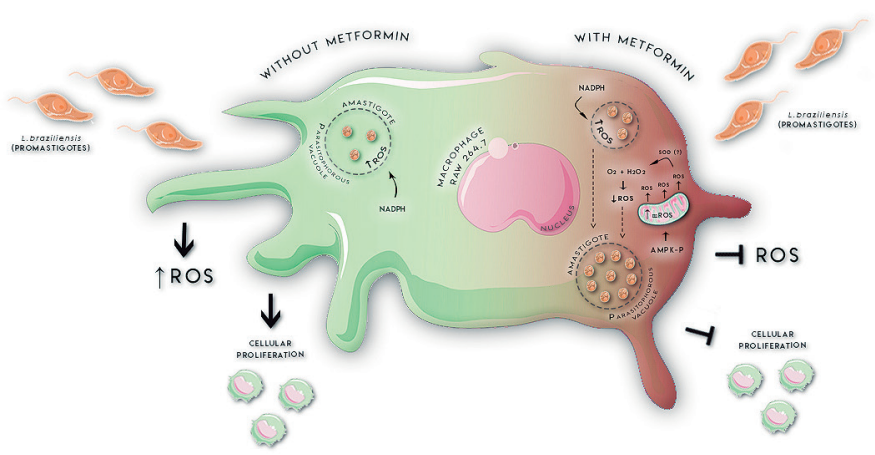

Fig. 6: proposed model of metformin-induced immunomodulation during infection by Leishmania braziliensis (Lb) in macrophages. Phagocytosis triggers the production of reactive oxygen species (ROS) via NADPH oxidase inside the parasitophorous vacuole. The absence metformin (MET) also does not interfere with the cellular proliferation and ensures greater control of parasitic load due to the absence of subversion of microbicidal macrophage mechanisms. Upon the introduction of MET, activated protein kinase (AMPK)-p is activated, suppresses cell proliferation and increases mROS. Phagocytosis induces ROS via NADPH oxidase, adding to the mROS generated via AMPK-p, providing an environment with high intracellular oxidative stress. This generated condition activates the superoxide dismutase (SOD) detoxification pathway of the host cell, as well as its isoforms in the parasite, reducing intracellular ROS and enabling parasitic growth inside the parasitophorous vacuole.

The pleiotropic effects of MET are also known to attenuate monocyte differentiation in macrophages, as well as inflammation-inducing events. ${ }^{(14)} \mathrm{We}$ found that $2 \mathrm{mM}$ MET inhibited the ability of macrophages to proliferate independent of the time of drug exposure, and did not interfere with cell viability. With regard to $L b$ growth, treatment with $2 \mathrm{mM}$ MET did not affect parasite growth, which remained in stationary growth phase, however this was not the case when using higher doses or the absence of MET. The Raw 264.7 macrophage cell line presents a doubling time of 16-24 $\mathrm{h} .{ }^{(23)}$ Accordingly, since MET did not modulate cell proliferation in 4-hour infection assays, this suggests that increased parasitic load and viability observed herein may be influenced by exposure to MET.

Wang et al. ${ }^{(24)}$ demonstrated the effects of MET in cancer, detailing that this drug inhibits abnormal cell proliferation by modifying the tumor microenvironment, which causes decreased angiogenesis, as well as by altering the tumor-associated macrophage phenotype that subsidises tumor development. ${ }^{(24)}$ In hepatocellular carcinoma, Del Campo et al. ${ }^{(25)}$ and Choi and Roberts ${ }^{(26)}$ corroborated findings reaffirming the direct action of MET in AMPK induction, mTOR inhibition, cell cycle suppression and the consequent proliferation of tumor cells.

Following our evaluations of the effect of MET in isolated macrophage cultures, MET was investigated in $L b$ infection in vitro. In addition to increased numbers of infected cells and parasitic load, in intracellular parasite viability was found to increase by $36 \%$ in MET treated macrophages within $96 \mathrm{~h}$ of infection compared to untreated infected cells. Considering that one of the main mechanisms of pathogen intracellular death is the production of ROS, ${ }^{(20)}$ and that MET interferes with the production of Mros, ${ }^{(12,17)}$ we hypothesise that, during the course of infection, $L b$ interferes with MET-induced ROS levels, which promotes increased parasite viability. In macrophage culture supernatants, we found that MET increases the release of ROS in uninfected cells; however, cells infected with $L b$ exhibited a significant decrease in ROS production following treatment with the drug.
The cultivation of uninfected macrophages with MET resulted in a $48.1 \%$ increase in mean intracellular ROS production, while stimulation by $L b$ infection promoted a 37.8\% greater synthesis compared to MET. Moreover, the introduction of MET in infected macrophage cultures was found to decrease intracellular ROS production by $34 \%$. Quintela-Carvalho et al. ${ }^{(27)}$ described that, in excess, ROS can be highly harmful to parasites and host cells. Therefore, to circumvent oxidative stress, host cells produce antioxidants, such as SOD-1, which catalyse the dismutation of superoxide in oxygen and hydrogen peroxide.(21) The mechanisms elucidated in the literature indicate that some parasites may induce antioxidant responses as an escape mechanism. In human macrophages, L. amazonensis infection is followed by dramatic increases in SOD-1 expression and activity, which has been shown to directly favor parasite survival. ${ }^{(27)}$

The data presented herein allow us to propose (Fig. 6) that the use of MET decreases cell proliferation in $L b$ infection and blocks the release of ROS into the extracellular medium. In addition, elevated production of ROS induced by Leishmania and the MET pathway, through the increase of AMPK-p, can generate high levels of cellular oxidative stress and promote the activation of host SOD production and its described isoforms in parasites, ${ }^{(28)}$ which will induce the detoxification of ROS and increase parasite load inside parasitophorous vacuoles.

Our experimental findings suggest that MET, the main hypoglycemic drug used in the treatment of diabetes, does not aid in the control of $L b$ infection, and may actually exacerbate viability and parasite load.

\section{ACKNOWLEDGEMENTS}

To all students and staff who participated in this project. The authors would like to thank Andris K Walter for assistance with manuscript copyediting and critical review. We are grateful to Joselia Cirqueira for pharmaceutical assistance.

\section{AUTHORS' CONTRIBUTION}

FRL - Substantially contributed to manuscript conception and design, acquisition of data, and/or analysis and interpreta- 
tion of data; FRL, LMF and TAM - authors participated in drafting and revision of the article; LMF, TAM, IBS and RL - contributed to the development of experiments; NMT and EMCF - conducted scientific guidance and advice; SA - author gave final approval of the final submitted version and any revisions, as well as provided supervision and orientation of the study. The authors declare that the research was conducted in the absence of any potential conflict of interest.

\section{REFERENCES}

1. Olga LSA, Santos JB. Advances in the treatment of cutaneous leishmaniasis in the New World in the last ten years: a systematic literature review. An Bras Dermatol. 2011; 86(3): 497-506.

2. MS/SVS/DVE - Ministério da Saúde/Secretaria de Vigilância em Saúde/Departamento de Vigilância Epidemiológica. Manual for surveillance of american integumentary leishmaniasis. Brasília: Ministério da Saúde; 2013.

3. Anversa L, Tiburcio MGS, Richini-Pereira VB, Ramirez LE, Anversa L, Tiburcio MGS, et al. Human leishmaniasis in Brazil: a general review. Rev Assoc Med Bra. 2018; 64(3): 281-9.

4. Carvalho EM. Editorial parasite, vectors and reservoirs as determinants of tegumentary leishmaniasis. Rev Soc Bras Med Trop. 2012; 45(4): 423-4.

5. Mougneau E, Glaichenhaus N. Cell biology and immunology of Leishmania. Immunol Rev. 2011; 240(1): 286-96.

6. Brito G, Dourado M, Polari L, Celestino D, Carvalho LP, Queiroz A, et al. Clinical and immunological outcome in cutaneous leishmaniasis patients treated with Pentoxifylline. Am J Trop Hyg. 2014; 90(4): 617-20.

7. Croft SL, Sundar S, Fairlamb AH. Drug resistance in leishmaniasis. Clin Microbiol Rev. 2006; 19(1): 111-26.

8. Tuon FF, Amato VS, Graf ME, Siqueira M, Nicodemo AC, Neto VA. Treatment of New World cutaneous leishmaniasis - a systematic review with a meta-analysis. Int J Dermatol. 2008; 47(2): $109-24$.

9. Gonzalez-Fajardo L, Fernández OL, Mcmahon-pratt D. Ex vivo host and parasite response to antileishmanial drugs and immunomodulators. PLos Negl Trop Dis. 2015; 9(5): 1-19.

10. Hattori Y, Hattori K, Hayashi T. Pleiotropic benefits of metformin: macrophage targeting its anti-inflammatory mechanisms. Diabetes. 2015; 64(6): 1907-9.

11. Kajiwara C, Kusaka Y, Kimura S, Yamaguchi T, Nanjo Y, Ishii $\mathrm{Y}$, et al. Metformin mediates protection against Legionella pneumonia through activation of AMPK and mitochondrial reactive oxygen species. J Immonol. 2018; 200(2): 623-31.

12. Boudy V, Daugan M, Dufa A. Metformin : an anti-diabetic drug to fight cancer. Pharmacol Res. 2016; 113(Pt A): 675-85.

13. Vasamsetti SB, Karnewar S, Kanugula AK. Metformin inhibits monocyte-to-macrophage differentiation via AMPK-mediated inhibition of STAT3 activation : potential role in atherosclerosis. Diabetes. 2015; 64(6): 2028-41.
14. Belkaid Y, Kamhawi S, Modi G, Valenzuela J, Noben-Trauth N, Rowton E, et al. Development of a natural model of cutaneous leishmaniasis: powerful effects of vector saliva and saliva preexposure on the long-term outcome of Leishmania major infection in the mouse ear dermis. J Exp Med. 1998; 188(10): 1941-53.

15. Qing L, Fu J, Wu P, Zhou Z, Yu F, Tang J. Metformin induces the $\mathrm{m} 2$ macrophage polarization to accelerate the wound healing via regulating ampk/mtor/nlrp3 inflammasome singling pathway. Am J Transl Res. 2019; 11(2): 655-68.

16. Ochoa-Gonzalez F, Cervantes-Villagrana AR, Fernandez-Ruiz JC, Nava-Ramirez HS, Hernandez-Correa AC, Enciso-Moreno JA, et al. Metformin induces cell cycle arrest, reduced proliferation, wound healing impairment in vivo and is associated to clinical outcomes in diabetic foot ulcer patients. PLoS One. 2016; 11(7): 1-16.

17. Singhal A, Jie L, Kumar P, Hong GS, Leow MKS, Paleja B, et al. Metformin as adjunct antituberculosis therapy. Sci Transl Med. 2014; 6(263): 263ra159.

18. Bridges HR, Jones AJY, Pollak MN, Hirst J. Effects of metformin and other biguanides on oxidative phosphorylation in mitochondria. Biochem J. 2014; 462(Pt3): 475-87.

19. Miyakoda M, Bayarsaikhan G, Kimura D, Akbari M, Udono H, Yui K. Metformin promotes the protection of mice infected with Plasmodium yoelii independently of $\gamma \delta \mathrm{T}$ cell expansion. Front Immunol. 2018; 9: 1-15.

20. Novais FO, Nguyen BT, Beiting DP, Carvalho LP, Glennie ND, Passos S, et al. Human classical monocytes control the intracellular stage of Leishmania braziliensis by reactive oxygen species. J Infect Dis. 2014; 209(8): 1288-96.

21. Fukai T, Ushio-Fukai M. Superoxide dismutases: role in redox signaling, vascular function, and diseases. Antioxid Redox Signal. 2011; 15(6): 1583-606.

22. Khouri R, Bafica A, Silva MPP, Noronha A, Kolb J-P, Wietzerbin $\mathrm{J}$, et al. IFN- $\beta$ impairs superoxide-dependent parasite killing in human macrophages: evidence for a deleterious role of SOD1 in cutaneous leishmaniasis. J Immunol. 2009; 182(4): 2525-31.

23. Raschke WC, Baird S, Ralph P, Nakoinz I. Functional macrophag cell lines transformed by Abelson leukemia virus. Cell. 1978; 15(1): 261-7.

24. Wang JC, Sun X, Ma Q, Fu GF, Cong LL, Zhang H, et al. Metformin's antitumour and anti-angiogenic activities are mediated by skewing macrophage polarization. J Cell Mol Med. 2018; 22(8): 3825-36.

25. Del Campo JA, García-Valdecasas M, Gil-Gómez A, Rojas Á, Gallego P, Ampuero J, et al. Simvastatin and metformin inhibit cell growth in hepatitis $\mathrm{C}$ virus infected cells via mTOR increasing PTEN and autophagy. PLoS One. 2018; 13(1): 1-13.

26. Choi J, Roberts LR. Statins and metformin for chemoprevention of hepatocellular carcinoma. Clin Liver Dis. 2016; 8(2): 48-52.

27. Quintela-Carvalho G, Luz NF, Celes FS, Zanette DL, Andrade D, Menezes D, et al. Heme drives oxidative stress-associated cell death in human neutrophils infected with Leishmania infantum. Front Immunol. 2017; 8(1620): 1-12.

28. Ghosh S, Goswami S, Adhya S. Role of superoxide dismutase in survival of Leishmania within the macrophage. Biochem J. 2003; 369(Pt 3): 447-52. 